\title{
Uso de estatinas e o risco de Diabetes Mellitus tipo 2: Revisão Baseada
} na Evidência

\author{
Statin Drug Use and the Risk of type 2 Diabetes Mellitus: Evidence-based Revision \\ Uso de estatinas y el riesgo de Diabetes Mellitus tipo 2: Revisión basada en evidencias \\ Susana Pires Silva. Unidade de Saúde Familiar (USF) Locomotiva, ACES Médio Tejo. Entroncamento. Portugal. \\ sm.pires.silvamail.com (Autora correspondente) \\ Nuno Monteiro. Unidade de Saúde Familiar (USF) D. Francisco de Almeida, ACES Médio Tejo. Abrantes. Portugal. \\ nunocavmonteiro@gmail.com
}

\section{Resumo}

Objetivo: Determinar se existe relação entre o uso de estatinas e a incidência de Diabetes Melittus tipo 2 (DM2). Métodos: Foram realizadas pesquisas nas bases de dados National Guidelines Clearinghouse, Cochrane Lybrary, Trip database, PubMed e MedLine utilizando os termos MeSH "Diabetes Mellitus Type 2" e "Hydroxymethylglutaryl-CoA Reductase Inhibitors" (inibidores da HMG-CoA redutase). Foram incluídas revisões sistemáticas e meta-análises e ensaios clínicos aleatorizados e controlados que avaliassem a incidência de DM2 em utilizadores de estatinas, quando comparado com placebo. Resultados: Foram selecionados 10 artigos (1 meta-análise e 9 ensaios clínicos aleatorizados e controlados). A evidência sugere que o uso de estatinas está associado a um aumento da incidência de DM2, principalmente com doses elevadas, em relação ao placebo, na maioria dos estudos avaliados. Conclusão: 0 risco de incidência de DM2 deve ser considerado, especialmente quando da prescrição de estatinas a indivíduos com baixo risco cardiovascular (SOR A), embora os estudos selecionados apresentem algumas limitações (metodologia heterogênea, sendo a maioria observacional).

\section{Abstract}

Objective: To investigate the relationship between statin administration and the incidence of Diabetes Mellitus type 2. Methods: A systematic review of the literature was conducted using the following databases National Guidelines Clearinghouse, Cochrane Lybrary, Trip database, PubMed and MedLine using the MeSH terms "Diabetes Mellitus Type 2" and "Hydroxymethylglutaryl-CoA Reductase Inhibitors". Systematic reviews, meta-analyses and randomized controlled trials analyzing the incidence of type 2 diabetes in statin users compared with placebo were included. Results: We selected 10 articles (1 meta-analysis and 9 randomized controlled trials). Evidence suggests that the use of statins is associated with an increased incidence of type 2 diabetes compared to placebo in most studies, especially at high dosages. Conclusion: The risk of incidence of type 2 diabetes should be considered especially when prescribing statins to patients with low cardiovascular risk (SOR A), despite the fact that selected studies present some limitations (heterogeneous methodology, and mostly observational).

\section{Palavras-chave:}

Diabetes Mellitus, Tipo 2

Inibidores de

Hidroximetilglutaril-CoA

Redutases

Incidência

Keywords:

Diabetes Mellitus, Type 2

Hydroxymethylglutaryl-CoA

Reductase Inhibitors

Incidence
Como citar: Silva SP, Monteiro N. Uso de estatinas e o risco de Diabetes Mellitus tipo 2: Revisão Baseada na Evidência. Rev Bras Med Fam Comunidade. 2016;11(38):1-8. http://dx.doi.org/10.5712/rbmfc10(38)1178
Fonte de financiamento: declaram não haver. Parecer CEP: não se aplica. Conflito de interesses: declaram não haver. Procedência e revisão por pares: revisado por pares. Recebido em: 05/07/2015. Aprovado em: 25/04/2016. 


\section{Resumen}

Objetivo: Determinar la relación entre el uso de estatinas y la incidencia de Diabetes Mellitus tipo 2. Métodos: Se realizaron encuestas en las bases de datos de National Guidelines Clearinghouse, Cochrane Lybrary, Trip database, PubMed y MedLine utilizando los términos MeSH "diabetes mellitus type 2" y "Hidroximetilglutaril-CoA reductase Innibitors". Se incluyeron revisiones sistemáticas, meta-análisis y ensayos controlados aleatorios para evaluar la incidencia de diabetes tipo 2 en los usuarios de estatinas en comparación con el placebo. Resultados: Se seleccionaron 10 artículos (1 meta-análisis y 9 ensayos controlados aleatorios). La evidencia sugiere que el uso de estatinas está asociado con un aumento de la incidencia de la diabetes tipo 2, especialmente con dosis más altas, en comparación con el placebo en la mayoría de los estudios evaluados. Conclusión: El riesgo de incidencia de diabetes tipo 2 debe considerarse especialmente al prescribir estatinas a los pacientes con riesgo cardiovascular bajo (SOR A), aunque los estudios seleccionados presentan algunas limitaciones (metodología heterogénea, siendo la mayor parte de los estudios observacionales).
Palabras clave:

Diabetes Mellitus, Tipo 2

Inhibidores de

Hidroximetilglutaril-CoA

Reductasas

Incidencia

\section{Introdução}

Vários ensaios clínicos e meta-análises demonstraram o benefício do uso de inibidores da HMG-CoA redutase na prevenção secundária de eventos cardiovasculares $(\mathrm{CV})$, mas a sua utilização em doentes com baixo risco $\mathrm{CV}$ e sem doença associada é mais controversa.

Com a prescrição tão difundida das estatinas, surgem riscos associados a esta classe farmacológica. Um risco emergente é o aumento da incidência de Diabetes Mellitus tipo 2 (DM2). Uma meta-análise recente sugere que existem fatores de risco metabólicos preexistentes que podem condicionar a incidência de DM2 em doentes medicados com estatinas. ${ }^{1}$ Não está claro se este risco varia com as diferentes estatinas ou se este é um efeito de classe e/ou relacionado com a dosagem. ${ }^{2,3}$ Embora alguns estudos indiquem que as estatinas atuam de forma diferente na homeostase da glicose em função de lipofilicidade e/ou potência relativa de ação, outros resultados diferem desta premissa. ${ }^{3}$

Uma meta-análise recente que englobou 17 ensaios clínicos, realizada por Mills et al., ${ }^{4}$ mostrou um aumento de novos casos de DM2 em doentes medicados com estatinas, sendo este um efeito de classe (Odds Ratio [OR], 1,09; IC 95\%,1,02-1,16).

Com este trabalho, pretendemos rever a literatura existente de forma a determinar se existe relação entre o uso de estatinas e a incidência de DM2.

\section{Métodos}

Foi realizada uma pesquisa bibliográfica de artigos de revisão sistemática (RS), meta-análises (MA) e ensaios clínicos aleatorizados controlados (ECAC). A pesquisa foi limitada a artigos publicados entre janeiro de 2004 a março de 2015, em inglês, espanhol ou português. A pesquisa foi baseada no PICO (Patients Intervention Comparator Outcome) (Figura 1). Os termos MeSH utilizados foram "Diabetes Mellitus Type 2" e "Hydroxymethylglutaryl-CoA Reductase Inhibitors" nas bases de dados National Guidelines Clearinghouse, Cochrane Lybrary, Trip database, PubMed e MedLine. Para determinar quais os artigos a serem incluídos na revisão, os dois autores (SS e NM) verificaram de forma independente os títulos e abstracts. Todos os artigos potencialmente relevantes foram avaliados pela leitura do artigo completo. Para a concordância da seleção dos artigos foi utilizada a escala de Jadad e Schultz (Jadad 1996; Schultz, 1995). As discordâncias foram resolvidas por consenso. 


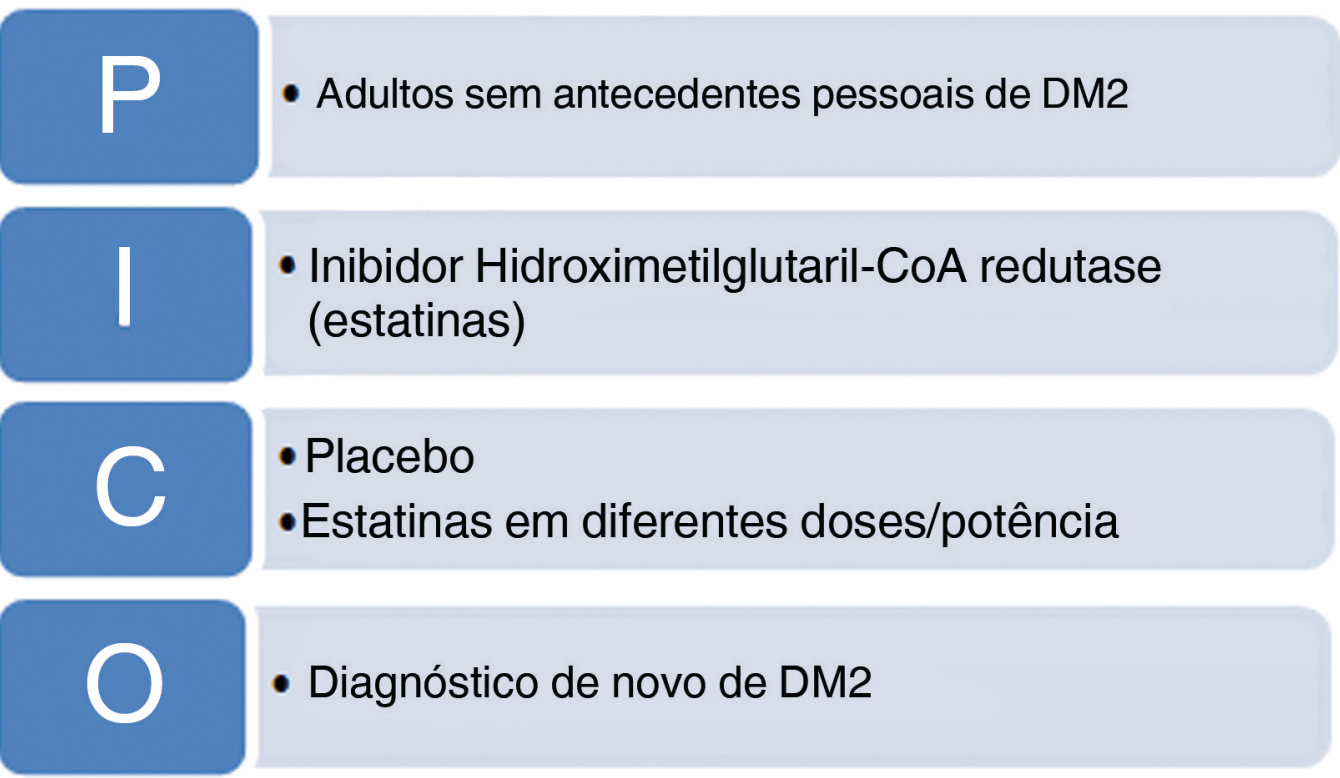

Figura 1. P: população; I: intervenção; C: controle; O: outcome/desfecho; DM2: Diabetes Mellitus tipo 2.

Os artigos selecionados para inclusão foram lidos integralmente pelos autores, que sistematizaram as informações em quadros estandardizados. Posteriormente, foram discutidos os conteúdos desses quadros para a atribuição de níveis de evidência aos estudos incluídos, bem como para produzir conclusões sumárias.

Os estudos incluídos foram avaliados com base na taxonomia Strength of Recommendation Taxonomy (SORT) da American Academy of Family Physicians. Essa escala avalia parâmetros tais como o tipo de estudo, a qualidade da amostra, a coerência dos resultados, evidências centradas no paciente versus na doença, permitindo a atribuição de níveis de evidência e força de recomendação finais. ${ }^{5}$

\section{Resultados}

Da pesquisa bibliográfica inicial resultaram 387 artigos, dos quais foram incluídos 10 (1 MA e 9 ECAC)-Figura 2.

A meta-análise elaborada por Navarese et al., ${ }^{6}$ que englobou 11 estudos incluindo 63.558 doentes, avaliou os novos casos de DM2 em doentes tratados com doses moderadas de estatinas ou placebo. Nos doentes tratados com estatinas em dose moderada ocorreram 2601 novos casos de DM2 num universo de 31.764 doentes $(8,18 \%)$ em comparação com 2527 novos casos entre 31.794 doentes (7,95\%) no grupo de controle-Quadro 1.

No ensaio desenvolvido por Jick e Bradbury, ${ }^{7}$ que envolveu uma população de 41.986 utentes medicados com estatinas e 27862 com placebo, foram identificados 588 novos casos de DM2 no grupo placebo e 2063 no grupo das estatinas. O Odds Ratio (OR) para desenvolver DM2 foi de 1,1 (IC 95\%, 0,8 -1,4)-Quadro 2.

No ensaio realizado por Culver et al., ${ }^{8}$ registaram-se 10.242 novos casos de DM2 por ano numa população de 1.004 .466 pessoas. O uso de estatinas estava associado a um aumento do risco de DM2 


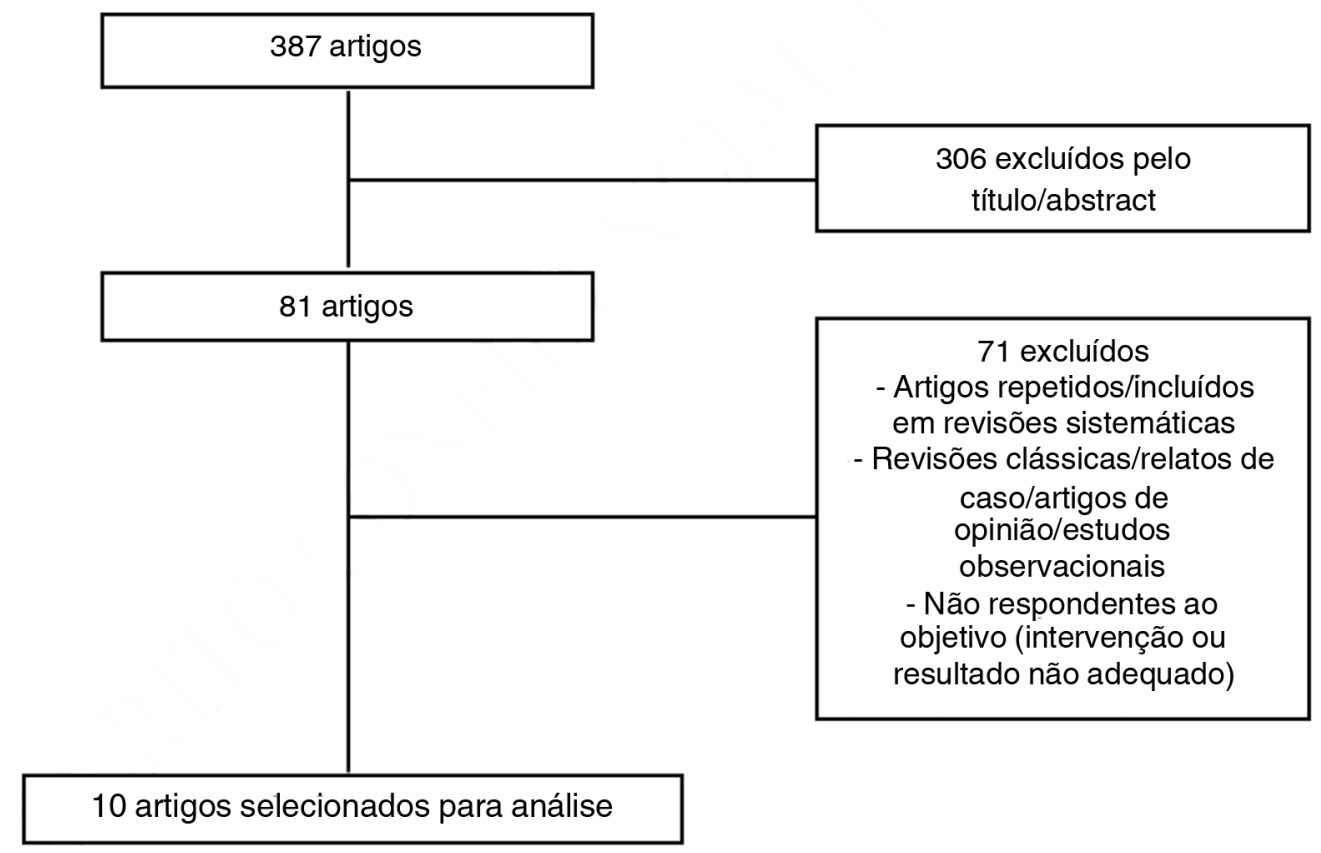

Figura 2. Organograma de seleção dos artigos para análise.

Quadro 1. Resumo das principais características, resultados e conclusões da revisão sistemática e atribuição de nível de evidência.

\begin{tabular}{|c|c|}
\hline \multicolumn{2}{|c|}{ Meta-Análise - NE 1} \\
\hline Navarese et al. 2013 & 17 ECAC, $n=113594$ (FU>1 ano) \\
\hline $\begin{array}{l}\text { 1. Elevada dose de estatina vs. placebo } \\
n=64137\end{array}$ & $\begin{array}{c}\text { - Rosuvastatina 20mg: associada } \uparrow 25 \% \text { do risco de incidência de } \\
\text { DM2 (OR: } 1,25, \text { IC } 95 \% 0,82-1,90) ; \\
\text { sinvastatina } 40 \mathrm{mg} \\
\text { - Pravastatina 40mg: } \downarrow 16 \% \text { risco incidência DM2 vs. outras } \\
\text { estatinas }\end{array}$ \\
\hline $\begin{array}{l}\text { 2. Moderada dose de estatina } \\
\mathrm{n}=63558\end{array}$ & $\begin{array}{l}\text { - Rosuvastatina 10mg: } \uparrow \text { risco incidência de DM2 (OR: } 1,11 \text {, IC } \\
95 \% \text { 0,81-1,53); } \\
\text { - Pravastatina 10-20mg: } \downarrow \text { risco DM2 vs. rosuvastatina }\end{array}$ \\
\hline $\begin{array}{l}\text { 3. Elevada dose de estatina vs. Moderada dose de estatina } \\
\mathrm{n}=23051\end{array}$ & $\begin{array}{l}\text { - Rosuvastatina: } 20 \mathrm{mg}>10 \mathrm{mg}: \uparrow 12 \% \text { incidência DM2 } \\
\text { - Pravastatina 40mg>10-20mg: } \uparrow 7 \% \text { incidência DM2 } \\
\text { - Atorvastatina alta dose } \approx \text { moderada dose }\end{array}$ \\
\hline
\end{tabular}

(HR1,71; IC 95\%, 1,61-1,83), quando comparado com o não uso. Esta associação diminuiu, mas permaneceu significativa após o ajuste para potenciais fatores de confundimento (HR 1,48; IC95\%, 1,38-1,59). Esta associação foi observada para todos os tipos de estatina e em diferentes doses-Quadro 2.

No ensaio de Wang et al., ${ }^{9}$ durante o período de acompanhamento médio de 7,2 anos, foram registados 5754 novos casos DM2. As curvas de Kaplan-Meier sugeriram um aumento do risco de incidência de DM2 nos utilizadores de estatinas (HR:1,15;IC 95\%:1,08-1,22; $p<0,001$ ). O tratamento com estatinas impediu um evento fatal em 202 indivíduos e conduziu a um novo caso de DM2 em 301 pacientes por ano-Quadro 2. No estudo de Carter et al., ${ }^{10}$ após o ajuste para fatores de confundimento, e em comparação com doentes tratados com pravastatina, aqueles tratados com atorvastatina, registraram um aumento de $22 \%$ no risco de DM2 (HR ajustado de 1,22; IC95\%;1,15-1,29). Os resultados sugerem que utentes idosos 
Quadro 2. Resumo das principais características, resultados e conclusões dos ECAC analisados e atribuição de níveis de evidência.

\begin{tabular}{|c|c|c|c|c|}
\hline \multicolumn{5}{|c|}{ ECAC } \\
\hline Referência & População & Intervenção & Resultados/Conclusões & NE \\
\hline $\begin{array}{l}\text { Jick, Bradbury. } \\
2004 \\
\text { Reino Unido }\end{array}$ & $\begin{array}{c}\text { Adultos } 30-79 \text { anos com } \\
\text { dislipidemia } \\
\text { n estatina }=69.348 \\
\text { FU } 11 \text { anos }\end{array}$ & Estatinas & $\begin{array}{c}\text { - Risco de DM2: nos indivíduos expostos (OR 1.1, IC } \\
\text { 95\%, 0,8-1,4) } \\
\text { - IMC>30 e HTA: favorece fatores independentes } \\
\text { associados ao risco de DM2 } \\
\text { - Sem relação entre a duração do tratamento e o risco } \\
\text { de DM2 }\end{array}$ & 2 \\
\hline $\begin{array}{l}\text { Culver et al. } \\
2012 \\
\text { EUA }\end{array}$ & $\begin{array}{l}\text { Mulheres em pós-meno- } \\
\text { pausa } \\
\text { (50-79 anos) } \\
\text { n=161.808 } \\
\text { FU } 9 \text { anos }\end{array}$ & $\begin{array}{l}\text { Estatinas de baixa e alta } \\
\text { potência/dose }\end{array}$ & $\begin{array}{l}\text { - Incidência novos casos de DM2: 9\% no grupo com } \\
\text { estatinas; } \\
\text { - Uso de estatinas aumenta o risco de incidência de } \\
\text { DM2 (HR 1,71, IC 95\%, 1,61-1,83); } \\
\text { - Associação estatisticamente significativa para } \\
\text { estatinas de alta e baixa dose/potencia. }\end{array}$ & 2 \\
\hline $\begin{array}{l}\text { Wang et al. } \\
2012 \\
\text { Taiwan }\end{array}$ & $\begin{array}{c}\text { Homens } \geq 45 \text { anos } \\
\text { Mulheres } \geq 50 \text { anos } \\
n=1 \text { milhão } \\
\text { FU } 12 \text { anos }\end{array}$ & Estatinas & $\begin{array}{c}\text { - Uso de estatinas aumenta o risco de incidência de } \\
\text { DM2 (HR 1,15, p<0,001) } \\
\text { - Analise risco-benefício favorece o uso de estatina em } \\
\text { alta dose em indivíduos de elevado risco cardiovascular } \\
\text { e em casos de prevenção secundária. }\end{array}$ & 1 \\
\hline $\begin{array}{l}\text { Carter et al. } \\
2013 \\
\text { Canadá }\end{array}$ & $\begin{array}{c}\text { Adultos }>66 \text { anos } \\
n=471.250 \\
\text { FU } 13 \text { anos }\end{array}$ & Estatinas & $\begin{array}{l}\text { - Indivíduos tratados com atorvastatina, rosuvastatina ou } \\
\text { sinvastatina: } \uparrow \text { risco de DM2 vs. pravastatina; } \\
\qquad \text { Incidência de DM2: } 10-22 \% \text {; } \\
\text { • Efeito dose-dependente: doses altas ou moderadas } \\
\text { levam a } \uparrow \text { risco de DM2 (HR 1.22, IC } 95 \% 1,19-1,26 \text { ) }\end{array}$ & 1 \\
\hline $\begin{array}{l}\text { Danaei et al. } \\
2013 \\
\text { Reino Unido }\end{array}$ & $\begin{array}{l}\text { Adultos de } 50-84 \text { anos } \\
\text { n=285.864 } \\
\text { FU } 10 \text { anos }\end{array}$ & Estatinas & $\begin{array}{l}\text { - Aumento do risco de incidência de DM2 no 1o ano de } \\
\text { FU (HR 1.24, IC 95\% 1,14-1,34) e após o 1ํano (HR } \\
\text { 1,12, IC 95\% 1,06-1,17). } \\
\text { - Atorvastatina: HR 1,22, IC 95\% 1,12-1,32 } \\
\text { - Sinvastatina: HR 1,14, IC 95\% 1,09-1,20 }\end{array}$ & 2 \\
\hline $\begin{array}{l}\text { Chen et al. } \\
2013 \\
\text { Taiwan }\end{array}$ & $\begin{array}{l}\text { Mulheres }>40 \text { anos } \\
\qquad \mathrm{n}=11.715 \\
\text { FU } 2 \text { anos }\end{array}$ & Estatinas & $\begin{array}{l}\text { - Aumento de incidência de DM2 mais evidente entre os } \\
\text { 40-64 anos do que acima dos } 65 \text { anos. } \\
\text { Efeito dose-dependente }\end{array}$ & 2 \\
\hline $\begin{array}{l}\text { Ko et al. } \\
2013 \\
\text { Canadá }\end{array}$ & $\begin{array}{l}\text { Adultos com }>65 \text { anos } \\
\text { com EAM prévio } \\
n=17.080 \\
\text { FU } 5 \text { anos }\end{array}$ & $\begin{array}{l}\text { Estatinas de elevada e } \\
\text { moderada potência/dose }\end{array}$ & $\begin{array}{c}\text { • Risco de DM2 de novo: } \\
\text { - Grupo com dose moderada } 13,0 \% \text {, Grupo com dose } \\
\text { elevada } 13,6 \% \\
\text { - Eficazes na } \downarrow \text { taxa de hospitalização pós-EAM }\end{array}$ & 1 \\
\hline $\begin{array}{l}\text { Dormuth et al. } \\
2014 \\
\text { Canadá, EUA, } \\
\text { Reino Unido }\end{array}$ & $\begin{array}{l}\text { Adultos } \geq 40 \text { anos } \\
\text { Prevenção secundária } \\
n=136.966 \\
\text { FU } 14 \text { anos }\end{array}$ & $\begin{array}{l}\text { Estatinas de elevada e } \\
\text { moderada potência/dose }\end{array}$ & $\begin{array}{l}\text { - Aumento do risco de DM2 de novo com as estatinas de } \\
\text { elevada potência nos primeiros } 4 \text { meses }(26 \%) \\
\text { • RR } 1,15,(\text { IC } 95 \%, 1,05-1,26)\end{array}$ & 2 \\
\hline $\begin{array}{l}\text { Macedo et al. } \\
2014 \\
\text { Reino Unido }\end{array}$ & $\begin{array}{l}\text { Adultos } 30-85 \text { anos } \\
\qquad \begin{array}{l}n=430.890 \\
\text { FU } 5 \text { anos }\end{array}\end{array}$ & Estatinas & $\begin{array}{l}\text { • Aumento do risco de DM2 (HR 1,57, IC 95\%, } \\
\text { 1,54-1,59), que aumenta com a duração da exposição }\end{array}$ & 2 \\
\hline
\end{tabular}

FU: Follow-up.

tratados com algumas estatinas têm risco aumentado para a incidência de diabetes, independentemente da dose ou se o tratamento é utilizado para a prevenção primária ou secundária. O risco parece ser maior com atorvastatina, rosuvastatina e sinvastatina. No entanto, após ajuste para a dose, o risco não parece persistir entre usuários de rosuvastatina-Quadro 2.

Os resultados encontrados no estudo de Danaei et al. ${ }^{11}$ mostram que os utilizadores de estatinas tiveram um aumento da incidência de DM2 em 14\%, em comparação com não utilizadores na população 
do Reino Unido. Em 1.170.000 pessoas por ano em seguimento, ocorreram 13.455 novos casos de DM2 e 8932 mortes. Estatinas lipofílicas (isto é, sinvastatina e atorvastatina) foram associadas a um risco aumentado de diabetes. O HR foi de 1,14 (IC 95\%, 1,09-1,20) para a sinvastatina e 1,22 (IC 95\%, 1,121,32) para a atorvastatina-Quadro 2.

O estudo de Chen et al. ${ }^{12}$ mostrou uma relação inversa entre o risco de novos casos de DM2 e a idade. Após o ajuste para outros fatores, o aumento da idade foi associado a uma diminuição do risco de DM2. Em indivíduos com idade 65-74 anos e mais de 75 anos ( $p=0,001, p<0,001$, respetivamente), em comparação com aqueles com idade entre 40-54 anos, o risco de DM2 foi reduzido em $28 \%$ e $37 \%$, respetivamente. Estes resultados ainda não foram explicados - Quadro 2.

No estudo de Ko et al. ${ }^{13}$, que teve uma duração de 5 anos, após a hospitalização de doentes com enfarte agudo do miocárdio (EAM), os que receberam estatinas em dose intensiva 13,6\% desenvolveram DM2 e ocorreram 13,0\% novos casos de DM2 nos doentes tratados com estatinas em dose moderada. $O$ aumento da incidência da DM2 associada a estatinas de dose intensiva foi de $0,3 \%$ em 1 ano (2,6\% no grupo de dose intensiva versus $2,3 \%$ no grupo de dose moderada) e maior em 4 anos, altura em que a diferença absoluta foi de $1,0 \%$ (11,7\% no grupo de dose intensiva versus $10,7 \%$ no grupo de dose moderada). No entanto, essa diferença não é significativamente estatística $(p=0,18)$ - Quadro 2.

No estudo de Dormuth et al. ${ }^{14}$, observou-se uma incidência de 3629 novos casos de DM2 nos primeiros dois anos de follow-up numa população de 136.966 pacientes. Observou-se uma incidência de DM2 de $15 \%$ em doentes a tomar uma dose intensiva/elevada de estatina em comparação com os doentes ao tomar estatina em baixa dose (HR 1,15, IC 95\%,1,05-1,26). Foi estimado que em 342 doentes medicados com estatina em dose elevada para prevenção secundária ocorreu um novo diagnóstico de DM2. O aumento do risco parece ser maior nos primeiros quatro meses de uso de estatina - Quadro 2.

No estudo de Macedo et al., ${ }^{15}$ durante o seguimento 130.395 indivíduos desenvolveram DM2, 56.702 casos entre utilizadores de estatina e 73.693 entre os do grupo placebo (sendo que no estudo existiam 3,8 pacientes no grupo placebo vs. 1 no grupo das estatinas). Os pacientes que desenvolveram DM2 eram mais velhos, com excesso de peso $\left(78,22 \%\right.$ com IMC> $\left.25 \mathrm{~kg} / \mathrm{m}^{2}\right)$ e mostraram-se mais propensos a ter comorbilidades, principalmente hipertensão e doenças cardiovasculares. Foi observado um risco aumentado de DM2 associado ao uso de estatinas. Este risco aumenta com a duração da utilização de estatinas, com IMC>25 kg/m² e diminui com a idade - Quadro 2.

\section{Discussão}

Algumas explicações para o aumento do risco de DM2 observado em doentes medicados com estatina incluem alterações na sinalização de insulina em tecidos periféricos, exacerbando a resistência à insulina, e/ou interação com a função das células $\beta$ pancreáticas, produtoras de insulina, prejudicando a sua secreção. ${ }^{16}$ Outro fator que pode influenciar o risco de DM2 com o uso de estatinas é o fato de estas reduzirem consideravelmente o colesterol. Em alguns estudos não existe ocultação aos doentes sobre qual o tratamento instituído pelo que esta polarização pode levar a que os doentes com substancial redução de LDL não adotem medidas higiênico-dietéticas, assumindo estilos de vida mais sedentários, com aumento ponderal, o que pode conduzir ao desenvolvimento de DM2. 
O aumento da produção de derivados de plasma de lipoproteínas de baixa densidade (LDL), como resposta compensatória à inibição da síntese de colesterol, pode resultar em inflamação e oxidação no interior da célula $\beta$. Consequentemente, a integridade estrutural e funcional das células $\beta$ é comprometida, prejudicando a secreção de insulina como resultado da apoptose celular. ${ }^{17}$ As estatinas podem também inibir a produção de insulina mediada pelo cálcio e diminuir a expressão dos transportadores de glicose célula $\beta$ GLUT-2 e GLUT-4. Finalmente, as estatinas também interferem com a síntese de ubiquinona (CoQ10), o que poderá alterar independentemente a secreção de insulina. ${ }^{17,18} \mathrm{O}$ grau em que as estatinas estão envolvidas nos mecanismos de indução da diabetes é variável e algumas estatinas representam um maior risco que outras.

A progressão da pré-diabetes para DM2 tem sido extensivamente estudada, sendo que um IMC elevado, aumento ponderal, pressão arterial elevada, idade mais jovem e triglicerídeos elevados foram identificados como preditores de desenvolvimento de diabetes. ${ }^{19-21} \mathrm{O}$ risco aumentado de desenvolver DM2, com o uso de estatina, é mais difícil de separar do efeito de outros preditores de agravamento da função das células beta/diminuição da sensibilidade da insulina ao longo do tempo. A DM2 é uma doença multifatorial, com complexas interações entre vários fatores ambientais, comportamentais e genéticos, tornando a contribuição de cada fator difícil de avaliar.

O uso de estatinas está associado a um aumento na ocorrência de diabetes. No entanto, o benefício do tratamento com estatina suplanta o risco em indivíduos de alto risco cardiovascular e em casos de prevenção secundária. Contudo, deve efetuar-se uma vigilância contínua, devendo ser incorporada, no programa de cuidados destes doentes, a determinação da glicemia em jejum para otimizar a gestão de risco global.

\section{Limitações}

Em vários estudos não consta a informação individual dos participantes quanto à história familiar de diabetes, ao consumo de tabaco, adesão terapêutica, entre outros possíveis fatores de confundimento face aos resultados apresentados.

\section{Conclusão}

O risco de incidência de DM2 deve ser considerado, especialmente quando da prescrição de estatinas a indivíduos com baixo risco cardiovascular (SOR A), embora os estudos selecionados apresentem algumas limitações (metodologia heterogênea, sendo a maioria observacionais). São necessários, por isso, mais estudos, por tratar-se de um assunto de elevada importância para a prática clínica, que pode ter consequências imediatas, nomeadamente na redução da prescrição generalizada de estatinas.

\section{Referências}

1. Waters DD, Ho JE, DeMicco DA, Breazna A, Arsenault BJ, Wun CC, et al. Predictors of new-onset diabetes in patients treated with atorvastatin: results from 3 large randomized clinical trials. J Am Coll Cardiol. 2011;57(14):1535-45. DOI: http:// dx.doi.org/10.1016/j.jacc.2010.10.047

2. Sattar N, Preiss D, Murray HM, Welsh P, Buckley BM, de Craen AJ, et al. Statins and risk of incident diabetes: a collaborative meta-analysis of randomised statin trials. Lancet. 2010;375(9716):735-42. DOI: http://dx.doi.org/ 10.1007/s00125-0143409-3. 
3. Baker WL, Talati R, White CM, Coleman Cl. Differing effect of statins on insulin sensitivity in non-diabetics: a systematic review and meta-analysis. Diabetes Res Clin Pract.2010;87(1):98-107. DOI: http://dx.doi.org/10.1016/j.diabres.2009.10.008

4. Mills EJ, Wu P, Chong G, Ghement I, Singh S, Akl EA, et al. Efficacy and safety of statin treatment for cardiovascular disease: a network meta-analysis of 170,255 patients from 76 randomized trials. QJM. 2011;104(2):109-24. DOI: http:// dx.doi.org/10.1093/qjmed/hcq165

5. Ebell MH, Siwek J, Weiss BD, Woolf SH, Susman J, Ewigman B, et al. Strength of recommendation taxonomy (SORT): a patient-centered approach to grading evidence in the medical literature. Am Fam Physician. 2004;69(3):548-56. PMID: 14971837 DOI: http://dx.doi.org/10.3122/jabfm.17.1.59

6. Navarese EP, Buffon A, Andreotti F, Kozinski M, Welton N, Fabiszak T, et al. Meta-analysis of impact of different types and doses of statins on new-onset diabetes mellitus. Am J Cardiol. 2013;111(8):1123-30. DOI: http://dx.doi.org/10.1016/j. amjcard.2012.12.037

7. Jick SS, Bradbury BD. Statins and newly diagnosed diabetes. Br J Clin Pharmacol. 2004;58(3):303-9. DOI: http://dx.doi. org/10.1111/j.1365-2125.2004.02142.x

8. Culver AL, Ockene IS, Balasubramanian R, Olendzki BC, Sepavich DM, Wactawski-Wende J, et al. Statin use and risk of diabetes mellitus in postmenopausal women in the Women's Health Initiative. Arch Intern Med. 2012;172(2):144-52. DOI: http://dx.doi.org/10.1001/archinternmed.2011.625

9. Wang KL, Liu CJ, Chao TF, Huang CM, Wu CH, Chen SJ, et al. Statins, risk of diabetes, and implications on outcomes in the general population. J Am Coll Cardiol. 2012;60(14):1231-8. DOI: http://dx.doi.org/10.1016/j.jacc.2012.05.019

10. Carter AA, Gomes T, Camacho X, Juurlink DN, Shah BR, Mamdani MM. Risk of incident diabetes among patients treated with statins: population based study. BMJ. 2013;346:f2610. DOI: http://dx.doi.org/10.1136/bmj.f2610

11. Danaei G, García Rodríguez LA, Fernandez Cantero O, Hernán MA. Statins and risk of diabetes: an analysis of electronic medical records to evaluate possible bias due to differential survival. Diabetes Care. 2013;36(5):1236-40. DOI: http://dx.doi. org/10.2337/dc12-1756

12. Chen CW, Chen TC, Huang KY, Chou P, Chen PF, Lee CC. Differential impact of statin on new-onset diabetes in different age groups: a population-based case-control study in women from an asian country. PLoS One. 2013;8(8):e71817. DOI: http://dx.doi.org/10.1371/journal.pone.0071817

13. Ko DT, Wijeysundera HC, Jackevicius CA, Yousef A, Wang J, Tu JV. Diabetes mellitus and cardiovascular events in older patients with myocardial infarction prescribed intensive-dose and moderate-dose statins. Circ Cardiovasc Qual Outcomes. 2013;6(3):315-22. DOI: http://dx.doi.org/10.1161/CIRCOUTCOMES.111.000015

14. Dormuth CR, Filion KB, Paterson JM, James MT, Teare GF, Raymond CB, et al.; Canadian Network for Observational Drug Effect Studies Investigators. Higher potency statins and the risk of new diabetes: multicentre, observational study of administrative databases. BMJ. 2014;348:g3244. DOI: http://dx.doi.org/10.1136/bmj.g3244

15. Macedo AF, Douglas I, Smeeth L, Forbes H, Ebrahim S. Statins and the risk of type 2 diabetes mellitus: cohort study using the UK clinical practice pesearch datalink. BMC Cardiovasc Disord. 2014;14:85. DOI: http://dx.doi.org/10.1186/1471-226114-85

16. Koh KK, Lim S, Sakuma I, Quon MJ. Caveats to aggressive lowering of lipids by specific statins. Int J Cardiol. 2012;154(2):97-101. DOI: http://dx.doi.org/10.1016/j.jjcard.2011.09.002

17. Sampson UK, Linton MF, Fazio S. Are statins diabetogenic? Curr Opin Cardiol. 2011;26(4):342-7. DOI: http://dx.doi. org/10.4093/dmj.2013.37.6.415.

18. Goldfine AB. Statins: is it really time to reassess benefits and risks? N Engl J Med. 2012;366(19):1752-5. DOI: http://dx.doi. org/10.14496/dia.41040851167.17.

19. Pani LN, Nathan DM, Grant RW. Clinical predictors of disease progression and medication initiation in untreated patients with type 2 diabetes and A1C less than 7\%. Diabetes Care. 2008;31(3):386-90. DOI: http://dx.doi.org/10.2337/dc07-1934

20. Nichols GA, Hillier TA, Brown JB. Progression from newly acquired impaired fasting glusose to type 2 diabetes. Diabetes Care. 2007;30(2):228-33. DOI: http://dx.doi.org/10.2337/dc09-0896.

21. Fonseca VA. Defining and characterizing the progression of type 2 diabetes. Diabetes Care. 2009;32 Suppl 2:S151-6. DOI: http://dx.doi.org/10.2337/dc09-S301 